Digital Supplement 1: CEASE Champion and Clinical Nurse Education/Competency Packet

\title{
Champion Role
}

In order to implement the CEASE Bundle, champions are being recruited from the ICU/SDU nursing staff. The Champions will help promote the education of staff from the ICU/SDU. Champions will educate nursing staff on application of an evidence-based patient customized monitoring bundle.

\section{Champion Education}

Champion education includes the following:

- Read one evidence-based article selected from the literature: Sendelbach S, Funk M. Alarm fatigue: a patient safety concern. AACN Adv Crit Care. 2013: 24:378-386.

- CEASE Bundle

- CEASE Bundle education packet

- Monitor demo mode instructions

\section{Champion Competency}

Champions will demonstrate competency by successfully completing the CEASE Champion Competency Checklist.

\section{Clinical Nurse Role}

In order to implement the CEASE Bundle, clinical nurses from the ICU/SDU nursing staff will attend an education session provided by CEASE Bundle champions. Champions will educate nursing staff on application of an evidence-based patient customized monitoring bundle.

\section{Clinical Nurse Education}

Champion education will include the following:

- Read one evidence-based article selected from the literature: Sendelbach S, Funk M. Alarm fatigue: a patient safety concern. AACN Adv Crit Care. 2013: 24:378-386.

- CEASE Bundle

- CEASE Bundle education packet

\section{Clinical Nurse Competency}

Clinical nurses will demonstrate competency by successfully completing the CEASE Clinical Nurse Competency. 
the Sounds: A tool to help in the management of monitoring patients as recommended by the American Association of Critical-Care Nurses

\section{co comminichion}

- Communicate to your colleagues and monitor techs about procedures on and off the unit, rhythm changes, etc. that could affect a patient's vital signs.

- Suspend alarms when nurses provide patient care that may produce false alarms.

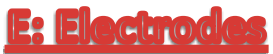

- Change ECG electrodes daily or more if needed

- Proper placement and skin preparation. Excessive hair should be clipped and skin should be clean and dried with a towel before electrode application.

- Change pulse oximetry sensors as needed.

- Check skin integrity under pulse oximetry sensor.

- Is ECG, SPO2, CVP, PAP, EtCO2, etc. monitoring clinically indicated for my patient? Is there an appropriate order?

- Team collaborative efforts can help determine appropriate monitoring and parameters.

- Discontinuing monitoring devices from patients, when monitoring parameters are no longer necessary.

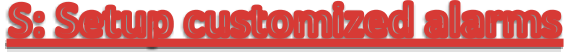

- Customizing alarm parameters to meet the needs of individual patients.

- Set customized alarms within 1 hour of assuming care of a patient and as patient's condition changes.

\section{Fip Finnerion}

- Education on the monitoring systems and alarm communication algorithms.

References: Christensen, et al., 2014; Clochesy, et al., 1991; Cvach, et al., 2013; Drew, et al., 2004; Feder \& Funk, 2013; Funk, et al. 2015; Graham \& Cvach, 2010; Görges, et al., 2009; HTF, 2011; Medina, et al., 1989; Sowan, et al., 2015; Whalen, et al., 2014 


\section{CEASE BUNDLE CHAMPION/CLINICAL NURSE EDUCATION PACKET}

\section{Communication}

- Important phone numbers: ICU tele tech: \& Hospital tele tech:

- Consider using the pause and silence button on the monitor for cares that affect vital signs. Button will pause alarm for 3 minutes.

- Place monitor on standby when taking patient off monitor for approved cares.

- Everyone is responsible for answering all alarms. Alert primary RN if you answer an alarm and do an intervention.

- Phones are an asset to communication.

\section{Electrodes}

- Change ECG electrodes daily or more if needed

- Proper placement and skin preparation. Excessive hair should be clipped and skin should be clean and dried with a towel before electrode application. Studies have shown a decrease in alarms by roughening up the skin before proper lead placement. Removes part of the outer layer of epidermis for greater conductivity

- Below is a picture of ideal lead placement. Respiratory rate monitoring depends on the lead placement for the RA and LL and how it examines the patient's rise and fall of the chest.

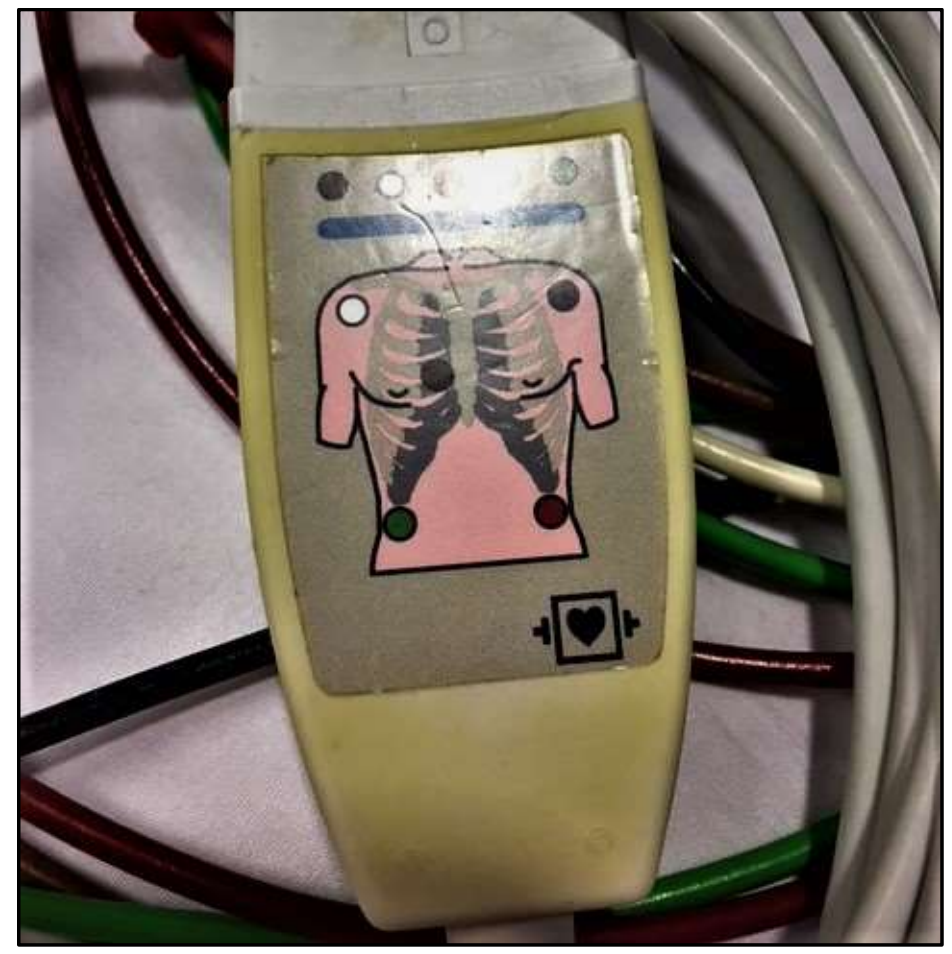

Photo: Provided with permission by Carmencita Lorenzo-Lewis 
- Change pulse oximetry sensors as needed. When the adhesive wears off, it is time for a new sensor.

- Check skin integrity under pulse oximetry sensor daily.

\section{Appropriate Actions}

- You are allowed to take off telemetry monitoring when a patient has orders for the floor without telemetry. You do not need to wait until the actual transfer of the patient. Also consider the necessity of other monitoring measures. Do you need continuous CVP or EtCO2 monitoring?

\section{Setup Customized Alarms}

- Examine patient's rhythm and vital signs to adequately assess alarms. (IE: Patient's $\mathrm{HR}$ is high. Are further interventions required for this? If not, consider changing alarms. If patient is has existing AFIB, consider turning off arrhythmia alarm.)

- When a patient's condition changes, change alarms to reflect needed changes.

- Consider changing certain alarms instead of turning off.

- Hot tip: When your art line is dampened but is used for blood draws, can change your alarms to lower value then the existing dampened mean. That way, if the patient pulls out their art line, it will alarm.

- Change alarms at central station.

- Activate patient in larger sector

- 2 ways to change alarms:

- Hover mouse over the vital sings values to activate box and change value

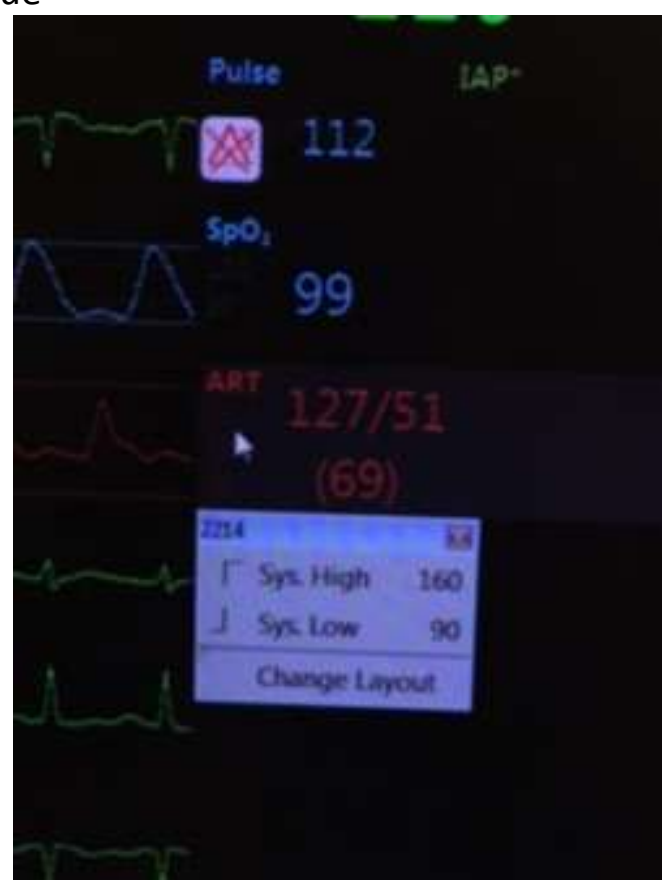

Photo: Provided with permission by Carmencita Lorenzo-Lewis 
- Click on measurements and specific vital sign to change

- Change alarms at monitor in room.

$\circ$ Touch the vital sign intended for change

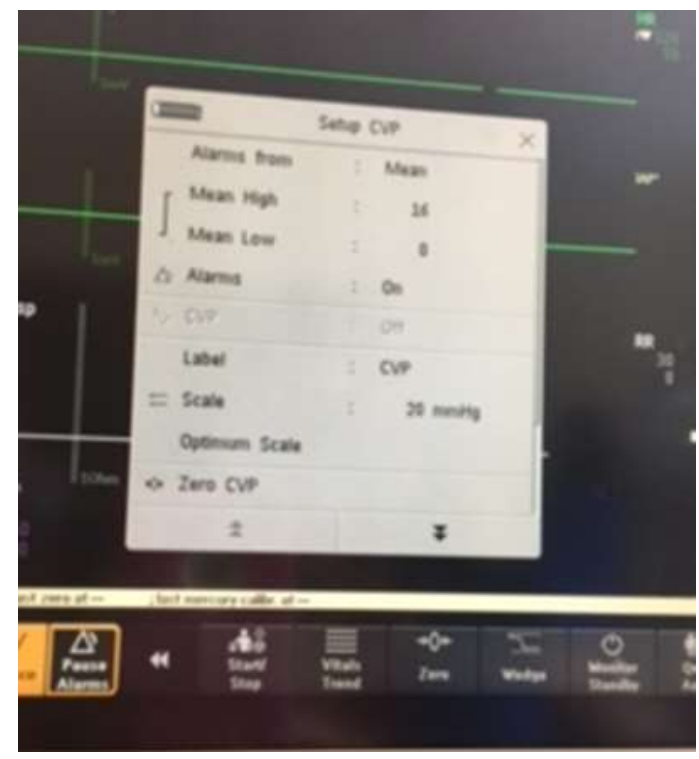

Photo: Provided with permission by Carmencita Lorenzo-Lewis

- Or touch module square at the bottom of the screen.

- This appears. Press on the intended vital sign to change the settings.

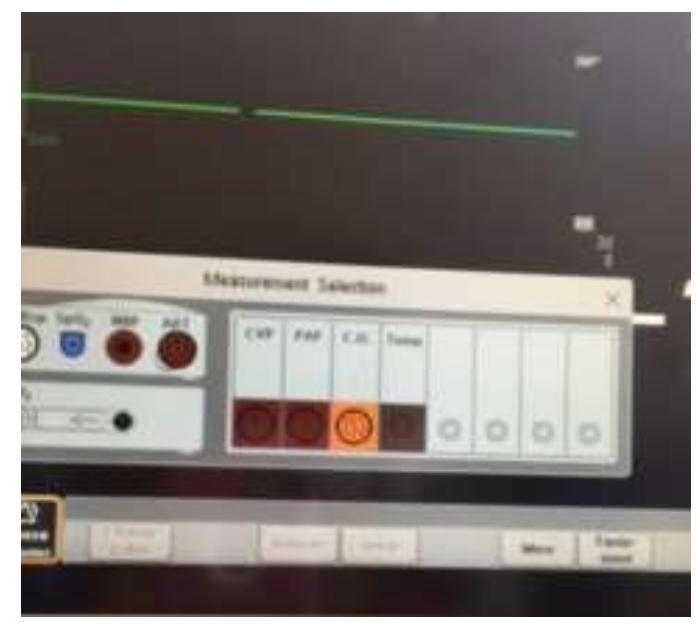

Photo: Provided with permission by Carmencita Lorenzo-Lewis 


\section{Additional Education}

- Review reading strips to examine patient's rhythm

- Review pairing rooms to monitor nurse about their patient while they are in the other room.

- Consider using the "relearning" function or changing the primary lead if monitor is having trouble tracking accurate rate.

\section{Sources of Evidence}

- American Association of Critical Care Nurses. Alarm management. AACN Practice Alert. Crit Care Nurs. 2013; 33(5):83-86.

- Cvach M, Biggs M, Rothwell K, Charles-Hudson C. (2013). Daily electrode change and effect on cardiac monitor alarms: an evidence-based practice approach. J Nurs Care Qual. 2013; 28:265-271.

- Drew B, Califf R, Funk M, et al. (2004). Practice standards for electrographic monitoring in hospital settings: an american heart association scientific statement form the councils on cardiovascular nursing, clinical cardiology, and cardiovascular disease in the young; endorsed by the international society of computerized electrocardiology and the american association of critical-care nurses. Circulation. 2004; 110:2721-2746.

- Monitoring system manufacturer's manual

- Sendelbach S, Funk M. (2013). Alarm fatigue: a patient safety concern. AACN Adv Crit Care. 2013; 24:378-386. 


\section{Monitor Demo Mode Education Sheet}

1. You can access the DEMO mode on any patient room monitor.

2. On the Smart Keys - push the button "Main Setup."

3. A pop up window appears labeled Main Setup

a. Scroll down on the pop up window to "Operating Modes."

b. Touch the Operating Modes line.

4. A new pop up window appears labeled "Operating Modes."

a. Touch the DEMO line.

b. Enter code xxxxx and then push enter.

5. The monitor is now in DEMO mode with simulated patient waveforms.

6. To return to patient monitoring

a. On the Smart Keys push "Main Setup."

b. Scroll down on the pop up window to "Operating Modes."

c. Touch the Operating Modes line.

d. Touch the Monitoring Line.

7. When complete, return the monitor to standby.

NOTE: ONLY the CEASE Champions received the monitor demo mode instructions during a hands-on educational session 